02

\title{
Анизотропия магниторезистивных свойств гранулярных высокотемпературных сверхпроводников как результат сжатия магнитного потока в межгранульной среде
}

\author{
(C) С.В. Семёнов ${ }^{1,2}$, Д.А. Балаев ${ }^{1,2}$, М.А. Почекутов ${ }^{1,2}$, Д.А. Великанов ${ }^{1}$ \\ ${ }^{1}$ Институт фризики им. Л.В. Киренского ФИЦ КНЦ СО РАН, \\ Красноярск, Россия \\ ${ }^{2}$ Сибирский фредеральный университет, \\ Красноярск, Россия \\ E-mail: svsemenov@iph.krasn.ru
}

(Поступила в Редакцию 16 ноября 2016 г.)

С целью выяснения причины известной анизотропии магниторезистивных свойств гранулярных высокотемпературных сверхпроводников (ВТСП), связанной со взаимной ориентацией магнитного поля Н и транспортного тока $\mathbf{j}$, исследовано поведение гистерезисных зависимостей магнитосопротивления $R(H)$ образца ВТСП иттриевой системы при перпендикулярной $(\mathbf{H} \perp \mathbf{j})$ и параллельной $(\mathbf{H} \| \mathbf{j})$ конфигурациях. На основании концепции эффективного поля в межгранульных границах (через которые туннелируют носители сверхпроводящего тока) проведен анализ гистерезисных зависимостей $R(H)$. Величина параметра, характеризующего эффективную степень сжатия магнитного потока в межгранульной среде, для перпендикулярной конфигурации оказалась примерно в 2 раза больше, чем для параллельной. Такой подход хорошо объясняет наблюдаемую многими авторами анизотропию магниторезистивных свойств гранулярных ВТСП (включая и данные по температурным зависимостям сопротивления в области резистивного перехода).

Исследование выполнено при финансовой поддержке РФФИ, Правительства Красноярского края, Краевого фонда наука в рамках научного проекта № 16-48-243018.

DOI: 10.21883/FTT.2017.07.44585.417

\section{1. Введение}

Известно, что гранулярные высокотемпературные сверхпроводники (ВТСП) демонстрируют анизотропию магнитосопротивления $R(H)$ и резистивного перехода $R(T)$ при различной взаимной ориентации магнитного поля Н и транспортного тока $\mathbf{j}$ (здесь и далее вектор $\mathbf{j}$ соответствует макроскопическому направлению тока, протекающего через образец) [1-13]. С одной стороны, это неудивительно, поскольку в классическом рассмотрении Бардина-Стефена для сверхпроводников второго рода зависящее от угла $\theta=\angle \mathbf{H}, \mathbf{j}$ магнитосопротивление пропорционально $\sin ^{2} \theta$ [14]. Скорость диссипации энергии на длину вихря зависит от произведения силы Лоренца на скорость движения вихря [14,15], а поскольку движение вихря возникает вследствие силы Лоренца, скорость вихря пропорциональна $\sin \theta$, что приводит к выражению $R \sim \sin ^{2} \theta$.

С другой стороны, наблюдаемая зависимость магнитосопротивления от угла $\theta$ для гранулярных ВТСП обусловлена процессами в хаотически ориентированных межгранульных границах, при этом сами ВТСПкристаллиты (гранулы) также ориентированы хаотически. Границы между ними представляют собой области с подавленными сверхпроводящими свойствами и проявляют себя как переходы джозефсоновского типа, через которые туннелируют носители сверхпроводящего тока. В такой хаотической системе причины наблюдаемой анизотропии магнитосопротивления требуют отдельного рассмотрения.

Некоторый прогресс в понимании причин такой анизотропии был достигнут в работе [7], в которой считалось, что магнитные поля, индуцированные экранирующими токами на поверхности сверхпроводящих гранул, вносят значительный вклад в величину магнитной индукции в среде между гранулами. И хотя авторы [7] получили хорошее согласие экспериментальных и модельных зависимостей величины $R(\mathbf{H} \| \mathbf{j}) / R(\mathbf{H} \perp \mathbf{j})$ от внешнего поля, тем не менее прямого определения эффективного поля в межгранульной среде в обсуждаемой работе проведено не было.

Методика определения эффективного поля в межгранульной среде и степени сжатия магнитного потока в межгранульных промежутках непосредственно из экспериментальных данных по намагничиванию и магнитосопротивлению была предложена в [16-18]. Целью настоящей работы является определение этих величин для случаев $\mathbf{H} \| \mathbf{j}$ и $\mathbf{H} \perp \mathbf{j}$, что позволит однозначно указать причину наблюдаемой анизотропии магниторезистивных свойств гранулярных ВТСП.

\section{2. Модель}

Рассмотрим схематично распределение в межгранульной среде локальных полей, индуцированных магнитными моментами сверхпроводящих гранул. Для двух 
гранул, отстоящих достаточно далеко друг от друга и находящихся во внешнем возрастаюшем поле $\left(H=H_{\uparrow}\right)$, будет наблюдаться картина распределения линий магнитной индукции $\mathbf{B}_{\text {ind }}$, схематично показанная на рис. $1, a . \mathbf{M}_{G}$ в данном рассмотрении - магнитный момент отдельной гранулы, который при $H=H_{\uparrow}$ направлен против внешнего поля, а распределение $\mathbf{B}_{\text {ind }}$ связано только с $\mathbf{M}_{G}$. В области вне гранулы на каждую точку действует локальное поле $\mathbf{B}_{\text {local }}(\mathbf{r})$, являющееся суперпозицией $\mathbf{B}_{\text {ind }}(\mathbf{r})$ и внешнего поля $\mathbf{H}$ :

$$
\mathbf{B}_{\text {local }}(\mathbf{r})=\mathbf{H}+\mathbf{B}_{\text {ind }}(\mathbf{r}) \text {. }
$$

Поскольку величина $\mathbf{B}_{\text {ind }}(\mathbf{r})$ связана с $\mathbf{M}_{G}$, можно записать

$$
\mathbf{B}_{\text {local }}(\mathbf{r})=4 \pi \mathbf{M}_{G} \mathbf{K}(\mathbf{r})+\mathbf{H},
$$

где $\mathbf{K}(\mathbf{r})$ зависит от геометрических параметров гранулы и распределения экранирующих токов и захваченного потока в ней.

Если „сдвинуть“ две гранулы близко (рис. 1, $b$ ) - до такой степени, как в поликристаллических материалах, то очевидно, что в области между двумя гранулами $A$ и $B$ будет иметь место значительное сгущение линий магнитной индукции $\mathbf{B}_{\text {ind. }}$ Тогда в интересующей нас области (между гранулами $A$ и $B$ на рис. $1, b$ ) можно ввести эффективное поле $\mathbf{B}_{\text {eff }}$, которое после усреднения по локальным полям (1) будет связано с магнитным моментом образца М следующим образом:

$$
\mathbf{B}_{\mathrm{eff}}=4 \pi \mathbf{M} \alpha+\mathbf{H} \text {. }
$$

Параметр $\alpha$ возникает при усреднении $\mathbf{K}(\mathbf{r})$ в выражении (1) и характеризует степень сгущения линий магнитной индукции, связанных с магнитным моментом гранул.

Выражение (2) объясняет происхождение гистерезиса магнитосопротивления $R(H)$ гранулярных ВТСП: $R \propto\left|B_{\text {eff }}\right|$ (если не вдаваться в детали функциональной зависимости $\left.R\left(B_{\text {eff }}\right)\right)$, а магнитный момент образца $M(H)$ сам является гистерезисной функцией внешнего поля (рис. 2). При возрастании внешнего поля $M\left(H_{\uparrow}\right)<0$. При убывании поля для $H_{\downarrow}=H_{\uparrow}$ имеет место неравенство $M\left(H_{\downarrow}\right)>M\left(H_{\uparrow}\right)$ либо значение $M\left(H_{\uparrow}\right)$ вообще положительно в некотором диапазоне полей (рис. 2). Если магнитный момент $M$ отрицателен $(M<0$ при $\left.H=H_{\uparrow}>0\right)$, то индуцированное поле в промежутке между гранулами $A$ и $B$ сонаправлено с внешним полем (рис. $1, b)$. Если $M>0$ (при $H=H_{\downarrow}>0$ ), то направление $\mathbf{B}_{\text {ind }}$ в области между гранулами $A$ и $B$ противоположно Н (стрелки, указывающие направления линий магнитной индукции на рис. $1, b$, изменят направление на противоположное). Следовательно, для эффективного поля в межгранульной среде, определяемого выражением (2), с учетом знака зависимости $M(H)$ получим

$$
\begin{aligned}
& B_{\text {eff }}\left(H_{\uparrow}\right)=-4 \pi M\left(H_{\uparrow}\right) \alpha+H_{\uparrow}, \\
& B_{\text {eff }}\left(H_{\downarrow}\right)=-4 \pi M\left(H_{\downarrow}\right) \alpha+H_{\downarrow} .
\end{aligned}
$$

Выражения (3),(4) позволяют определить степень сжатия магнитного потока - коэффициент $\alpha$, который, как правило, оказывается значительно (на порядок) больше единицы [16-19]. Для определения $\alpha$ используется достаточно очевидное условие: если для экспериментальной гистерезисной зависимости $R(H)$ выполняется $R\left(H_{\uparrow}\right)=R\left(H_{\downarrow}\right)$, то и $B_{\text {eff }}\left(H_{\uparrow}\right)=B_{\text {eff }}\left(H_{\downarrow}\right)$. Тогда из (3) и (4) получаем

$$
\Delta H=H_{\downarrow}-H_{\uparrow}=\alpha 4 \pi\left\{M\left(H_{\downarrow}\right)-M\left(H_{\uparrow}\right)\right\} .
$$

С помощью выражения (5) можно определить значение $\alpha$, используя экспериментальные гистерезисные
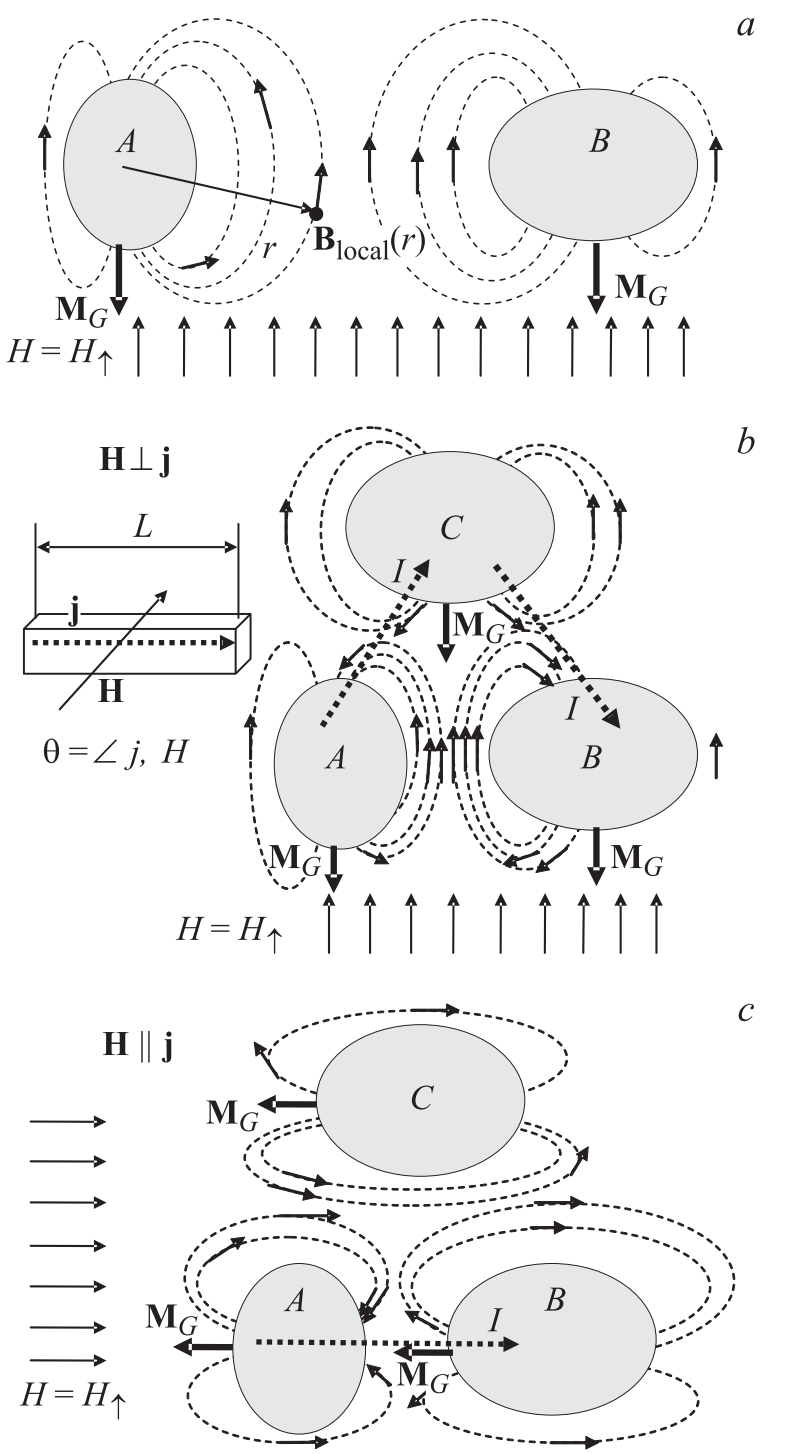

Рис. 1. Схематическое представление линий магнитной индукции в межгранульной среде, связанных с магнитными моментами сверхпроводящих гранул. $a-$ гранулы находятся достаточно далеко друг от друга; $b$ и $c-$ сгущение линий магнитной индукции при достаточно близком расположении гранул и схематические траектории микроскопических токов $I$ для перпендикулярной $(b)$ и параллельной $(c)$ конфигураций Н и ј. Для всех случаев внешнее поле возрастает: $H=H_{\uparrow}$. На части $b$ слева показана схема эксперимента. 


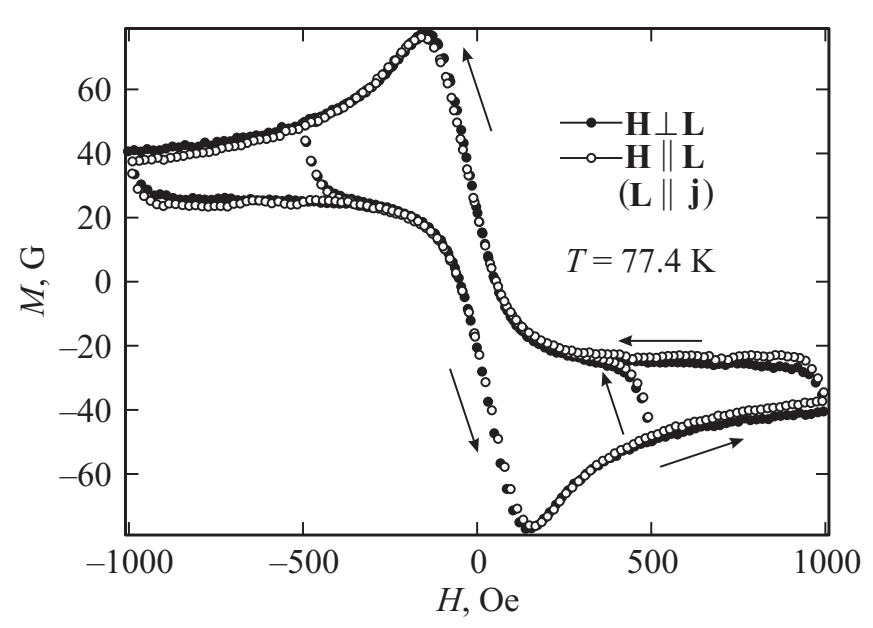

Рис. 2. Петли магнитного гистерезиса $M(H)$ исследованного образца при значениях максимального приложенного поля $H_{\text {app }}=500$ и 1000 Ое для указанных конфигураций (схема эксперимента приведена на рис. $1, b)$. Стрелки показывают направление изменения внешнего магнитного поля.

зависимости $R(H)$ и $M(H)$, полученные на одном и том же образце. Как оказалось, неплохим приближением можно считать, что $\alpha$ слабо зависит от магнитного поля, т.е. $\alpha \neq f(H)$, хотя в области полей, в которой $M \approx 0$ (при этом условии вклад захваченного магнитного потока и диамагнитный вклад примерно одинаковы), влияние магнитных моментов гранул остается весьма значимым.

Теперь перейдем к вопросу о протекании микроскопических токов I через межгранульные границы в случаях $\mathbf{H} \| \mathbf{j} \quad$ и $\quad \mathbf{H} \perp \mathbf{j}$. Для перпендикулярной ориентации вариант протекания тока от гранулы $A$ к грануле $B$ (рис. $1, b)$, очевидно, окажется невыгодным, поскольку

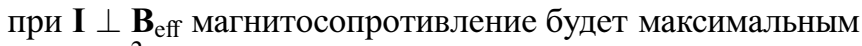
$\left(R \propto \sin ^{2} \theta, \theta=\angle \mathbf{H}, \mathbf{j}\right)$. Естественно, что для микроскопического транспортного тока будет более „выгоден“ путь через соседнюю гранулу $(C)$, при котором взаимная ориентация I и $B_{\text {eff }}$ будет близка к параллельной (т.е. путь $A \rightarrow C \rightarrow B$, схематично показанный на рис. $1, b)$. Если $\mathbf{H} \| \mathbf{j}$, то наиболее оптимальным путем протекания остается $A \rightarrow B$, как схематично показано на рис. $1, c$. Разумеется, что и в этом случае есть большое количество межгранульных границ, для которых нарушается параллельность Н и ј. Такое отличие от идеальной параллельной ориентации проявляется в том, что даже для этого случая $(\mathbf{H} \| \mathbf{j})$ величина коэффициента $\alpha$ оказывается порядка 10. В цитированных работах [1619] измерения были проведены именно для этой ориентации. Логично предположить, что для одного и того же образца значение $\alpha$ при $\mathbf{H} \perp \mathbf{j}$ будет больше, чем при $\mathbf{H} \| \mathbf{j}$. Экспериментальное доказательство этого окончательно подтвердит, что именно сгущение магнитного потока (значительная величина $\alpha$ ) определяет наблюдаемую анизотропию магнитосопротивления (магнитное поле-транспортный ток) гранулярных ВТСП.

\section{3. Эксперимент}

ВТСП $\mathrm{Y}_{0.98} \operatorname{Pr}_{0.02} \mathrm{Ba}_{2} \mathrm{Cu}_{3} \mathrm{O}_{7}$ был приготовлен по стандартной технологии твердофазного синтеза. Данные рентгеноструктурного анализа показали наличие рефлексов только от структуры 1-2-3. Для образца наблюдался типичный для таких систем металлический ход зависимости $R(T)$ выше $T_{C}(90.5 \mathrm{~K})$.

Транспортные измерения были проведены на образцах с типичным размером $\sim 1 \times 1 \times 7 \mathrm{~mm}$. Зависимости магнитосопротивления $R(H)=U(H) / j \quad(U-$ падение напряжения, $j$ - транспортный ток) и сопротивления от температуры $R(T)$ были измерены стандартным четырехзондовым методом. Транспортный ток протекал вдоль длинной части образца. Во время измерений зависимостей $R(H)$ (приведены данные для значения тока $j=30 \mathrm{~mA}$ ) образец находился в среде жидкого азота. Внешнее поле задавалось электромагнитом ФЛ-1, что позволяло измерять транспортные характеристики как для параллельной $(\mathbf{H} \| \mathbf{j})$, так и для перпендикулярной $(\mathbf{H} \perp \mathbf{j})$ ориентаций. После выполнения условий охлаждения в нулевом внешнем поле (ZFC) внешнее поле изменялось до значений максимального приложенного поля $H_{\text {арр }}= \pm 500$ или \pm 1000 Ое. Далее анализируются данные для прямой и обратной ветвей гистерезисных зависимостей (за исключением начального хода от $H=0$ до $H_{\text {app }}=+500$ Ое). Зависимости $R(T)$ были измерены как в условиях ZFC, так и при охлаждении во внешнем поле (FC) с последующим „выключением“ поля при двух ориентациях $(\mathbf{H} \| \mathbf{j}$ и $\mathbf{H} \perp \mathbf{j})$.

Магнитные измерения проводились на вибрационном магнитометре [20]. Для них готовились образцы с геометрическими параметрами, подобными таковым при транспортных измерениях $(\sim 0.5 \times 0.5 \times 3.5 \mathrm{~mm})$. Это позволяет считать, что размагничивающий фактор формы образца при магнитных и транспортных измерениях одинаков. Установка [20] на основе электромагнита конструкции Пузея позволяет проводить измерения как при $\mathbf{H} \| \mathbf{L}$, так и при $\mathbf{H} \perp \mathbf{L}(\mathbf{L}-$ вектор, направленный вдоль длинной стороны образца). Скорость изменения внешнего поля была одинаковой $(\sim 2 \mathrm{Oe} / \mathrm{s})$ как для транспортных, так и для магнитных измерений. Зависимости $M(H)$ и $M(T)$ были измерены при тех же внешних условиях, что и зависимости $R(T)$.

\section{4. Результаты и обсуждение}

4.1. Анизотропия магнитосопротивления $R(H)$. Для корректного сопоставления зависимостей $M(H)$ и $R(H)$ измерения магнитных свойств были проведены при двух взаимных ориентациях $\mathbf{L}$ (длинной стороны образца) и внешнего поля Н. Зависимости $M_{\perp}(H)$ $($ при $\mathbf{H} \perp \mathbf{L})$ и $M_{\|}(H)$ (при $\left.\mathbf{H} \| \mathbf{L}\right)$ для исследованного образца приведены на рис. 2. Как видно, различие между случаями $\mathbf{H} \| \mathbf{L}$ и $\mathbf{H} \perp \mathbf{L}$ незначительно (наблюдается небольшое расхождение зависимостей $M_{\perp}(H)$ и $M_{\|}(H)$ 
в области полей $500-1000 \mathrm{Oe})$, размагничивающий фактор формы образца не играет определяющей роли для магнитных измерений гранулярных ВТСП. Петля гистерезиса $M(H)$ типична для гранулярных ВТСП, синтезированных по стандартной технологии [21].

На рис. 3 приведены гистерезисные зависимости $R(H)$ для параллельной $R_{\|}(H)$ и перпендикулярной $R_{\perp}(H)$ взаимной ориентации $\mathbf{H}$ и ј. Обсуждаемая выше анизотропия магнитосопротивления $-R_{\perp}(H)>R_{\|}(H)-$ отчетливо видна на этом рисунке. Вставка на рис. 3 показывает поведение зависимостей $R(H)$ в диапазоне $\pm 55 \mathrm{Oe}$. Минимумы зависимостей $R\left(H_{\downarrow}\right)$ возникают при условии наибольшей компенсации внешним полем поля $\mathbf{B}_{\text {ind }}$, индуцированного магнитными моментами гранул. При значениях $H_{\downarrow}$, меньших $\sim 50$ Ое, величина $M\left(H_{\downarrow}\right)$ положительна (рис. 2) и члены в правой части выражения (4) имеют разные знаки, что приводит к появлению минимума зависимостей $B_{\text {eff }}\left(H_{\downarrow}\right)$ и $R\left(H_{\downarrow}\right)$.

Горизонтальные линии на рис. 3 показывают пример определения полевой ширины гистерезиса магнитосопротивления $\Delta H=\left|H_{\uparrow}-H_{\downarrow}\right|$ при одном и том же значении $H_{\uparrow}=90 \mathrm{Oе}$. Видно, что величина $\Delta H$ (разница между значениями полей $H_{\uparrow}$ и $H_{\downarrow}$, при которых горизонтальная прямая пересекает зависимости $R\left(H_{\downarrow}\right)$ ) для $R_{\perp}(H)$ заметно превышает $\Delta H$ зависимости $R_{\|}(H)$. Ранее установлено, что параметр $\Delta H$ (при одной выбранной ориентации $\mathbf{H}$ и $\mathbf{j}$ ) не зависит от транспортного тока в широком диапазоне этой величины [22-24].

Для определения значений $\alpha$ при параллельной и перпендикулярной ориентациях мы воспользовались данными магнитных измерений (рис. 2) и построили гистере-

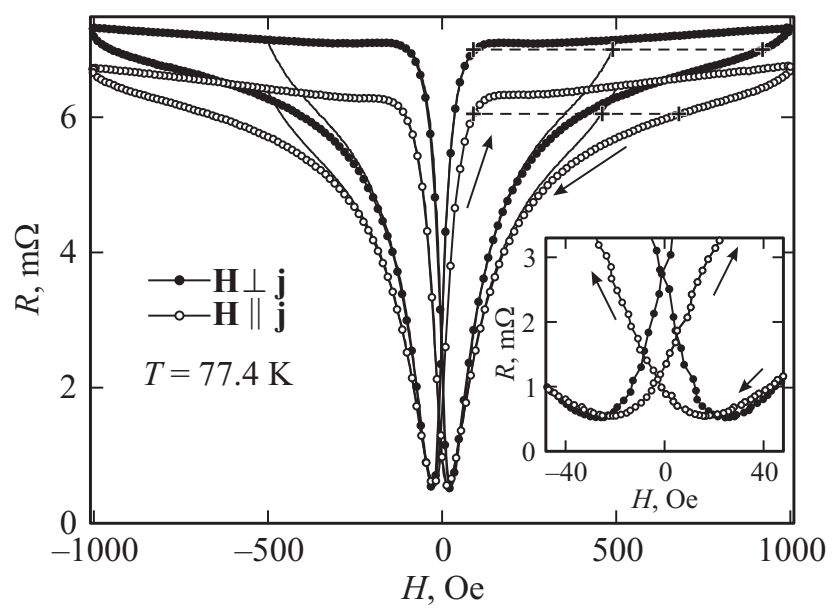

Рис. 3. Гистерезисные зависимости магнитосопротивления $R(H)$ исследованного образца при значениях максимального приложенного поля $H_{\text {app }}=500$ и 1000 Ое для указанных конфигураций (схема эксперимента приведена на рис. $1, b$ ). Стрелки показывают направление изменения внешнего магнитного поля. Сплошными линиями показан обратный ход зависимостей $R\left(H_{\downarrow}\right)$ для $H_{\text {app }}=500$ Ое. Горизонтальные линии показывают пример определения полевой ширины гистерезиса $\Delta H$ при значении $H_{\uparrow}=90$ Ое. На вставке - ход зависимостей $R(H)$ в области малых полей.

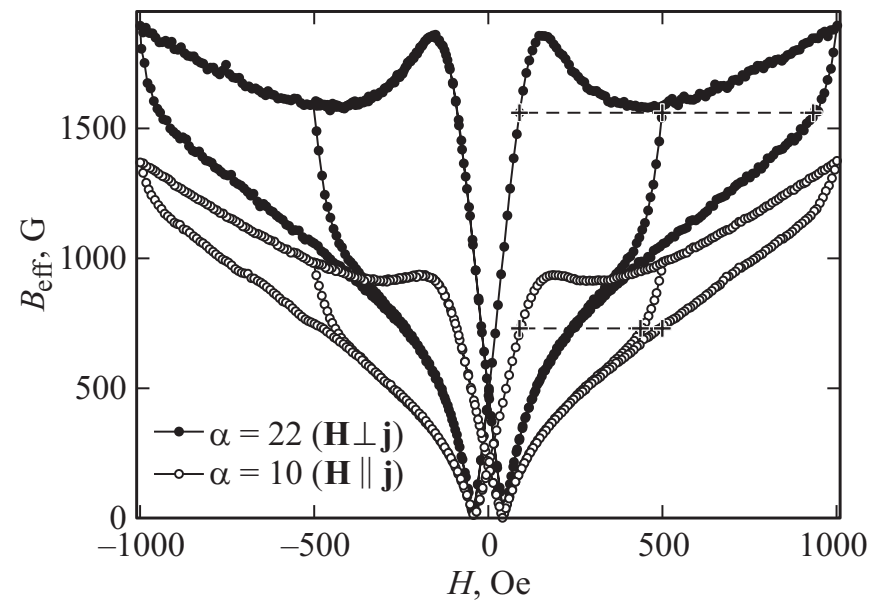

Рис. 4. Гистерезисные зависимости эффективного поля $B_{\mathrm{eff}}(H)$ в межгранульной среде (модуль выражений (3), (4)), полученные с использованием данных $M(H)$ (рис. 2) при двух значениях параметра $\alpha$ для перпендикулярной и параллельной конфигураций. Горизонтальные линии - пример определения полевой ширины гистерезиса $\Delta H$ при значении $H_{\uparrow}=90$ Ое (аналогично рис. 3).

зисные зависимости $B_{\text {eff }}(H)$ по выражениям (3) и (4). Поскольку магнитосопротивление определяется не направлением $B_{\text {eff, }}$ а модулем этой величины $-R \propto\left|B_{\text {eff }}\right|$, брался модуль функции $B_{\text {eff }}(H)$. Затем значения $\alpha$ подбирались такими, чтобы величина $\Delta H=H_{\downarrow}-H_{\uparrow}$, определяемая непосредственно из зависимостей $B_{\text {eff }}\left(H_{\uparrow}\right)$ и $B_{\text {eff }}\left(H_{\downarrow}\right)$, была близка к величине $\Delta H$, полученной из экспериментальных зависимостей $R(H)$ (рис. 3). При этом для сопоставления $R_{\perp}(H)$ и $R_{\|}(H)$ брались соответственно данные для $M_{\perp}(H)$ и $M_{\|}(H)$. Кроме того, делалось еще одно упрощение: в выражениях (3) и (4) не учитывались различные проекции вектора Н для ориентаций $\mathbf{H} \| \mathbf{j}$ и $\mathbf{H} \perp \mathbf{j}$. ${ }^{1}$

Оказалось, что наилучшее согласие для большого диапазона полей (см. далее) имеет место при значениях $\alpha$, равных $\sim 22-25$ для перпендикулярной и $\sim 10-15$ для параллельной конфигураций. Бо́льшие значения $\alpha$ лучше описывают данные для диапазона полей $500-100 \mathrm{Oe}$, и это, видимо, связано с тем, что условие $\alpha \neq f(H)$ выполняется только в первом приближении (см. раздел 2).

Рис. 4 иллюстрирует полученные зависимости $B_{\text {eff }}(H)$ для $\alpha=10$ и 22. Горизонтальные линии на этом рисунке - пример определения величины $\Delta H$ при $H_{\uparrow}=90 \mathrm{Oe}$ для этих конфигураций (подобно примеру, показанному на рис. 3 для зависимостей $R(H)$ ). Видно, что уменьшение $\Delta H$ для параллельной конфигурации, связанное с

\footnotetext{
${ }^{1}$ Очевидно, что для поликристалла угол $\theta$, входящий в выражение для проекции $\mathbf{H}$ на направление $\mathbf{j}(H \sin \theta)$, не будет строго равен 0 и $90^{\circ}$ для ориентаций $\mathbf{H} \| \mathbf{j}$ и $\mathbf{H} \perp \mathbf{j}$. Варьирование значений „эффективного“ угла $\theta$ для этих случаев приводит к появлению дополнительных подгоночных параметров, хотя, по нашим оценкам, и не влияет кардинально на сделанные выводы о большем значении параметра $\alpha$ для перпендикулярной конфигурации.
} 


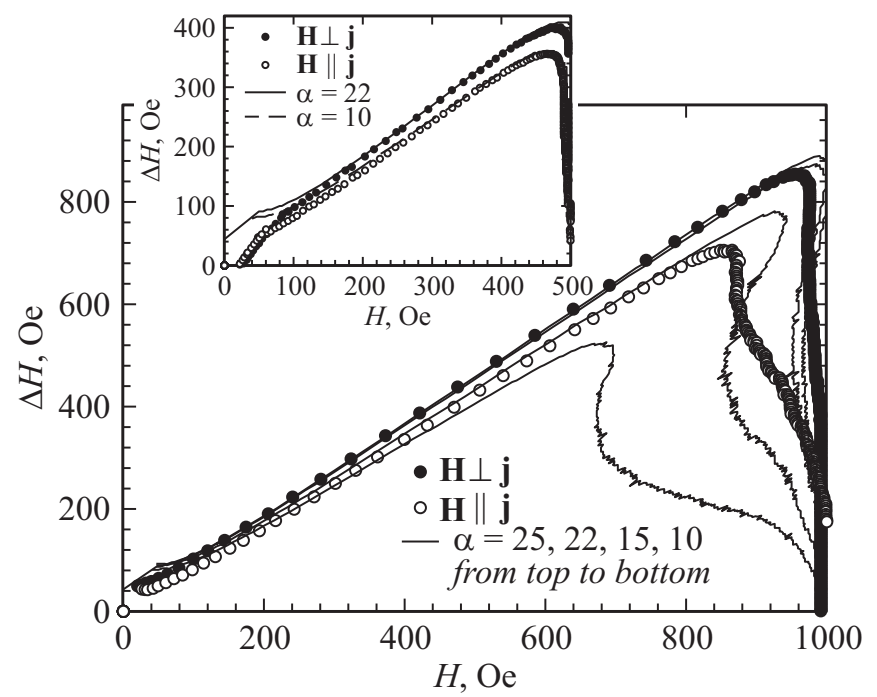

Рис. 5. Зависимости полевой ширины гистерезиса $\Delta H$ от $H_{\downarrow}$, полученные из зависимостей $R(H)$ (точки), а также из зависимостей $B_{\text {eff }}(H)$ по формулам (3), (4) (линии) при различных значениях параметра $\alpha$. Приведены данные для $H_{\text {app }}=1000$ Oe, на вставке - для $H_{\text {app }}=500 \mathrm{Oe}$.

меньшим значением параметра $\alpha$, хорошо воспроизводится при использованном подходе.

На рис. 5 приведены данные по зависимостям $\Delta H$ от $H_{\downarrow}$ (длина горизонтальных отрезков между $H_{\downarrow}$ и $H_{\uparrow}$ на рис. 3 и 4 при условии $R=$ const и $B_{\text {eff }}=$ const), которые были получены при максимальном приложенном поле $H_{\text {app }}=1000$ Ое из гистерезисных зависимостей $R(H)$ (точки) и $B_{\mathrm{eff}}(H)$ (линии). Расчетные зависимости $\Delta H\left(H_{\downarrow}\right)$ получены при нескольких значениях $\alpha$. Вставка на рис. 5 иллюстрирует данные, полученные при $H_{\text {app }}=500$ Ое. Несмотря на некоторый разброс значений $\alpha$ для диапазонов до 500 Ое и 500-1000 Ое, хорошее согласие между экспериментальными и расчетными зависимостями $\Delta H\left(H_{\downarrow}\right)$ позволяет утверждать, что для параллельной конфигурации $\mathbf{H} \| \mathbf{j}$ параметр $\alpha$ уменьшается примерно в 2 раза по сравнению со значением для случая $\mathbf{H} \perp \mathbf{j}$. Это подтверждает сделанные в разделе 2 предположения о характере сгущения линий магнитной индукции в межгранульной среде.

4.2. Анизотропия зави симостей $R(T)$. Дополнительным подтверждением отмеченного выше является влияние взаимной ориентации Н и $\mathbf{j}$ на резистивный переход. На рис. 6 представлены зависимости $R(T)$ для исследуемого образца в области резистивного перехода для случаев $\mathbf{H} \| \mathbf{j}$ и $\mathbf{H} \perp \mathbf{j}$. Резкий скачок сопротивления соответствует переходу в ВТСП-гранулах, а плавная часть зависимости $R(T)$ - переходу в подсистеме межгранульных границ [17,24-26]. Видно, что различие зависимостей $R(T)$ для случаев $\mathbf{H} \| \mathbf{j}$ и $\mathbf{H} \perp \mathbf{j}$ имеет место в температурном диапазоне, соответствующем резистивному переходу в межгранульной среде, что наблюдалось ранее $[7,10]$.
Применим описанный выше подход для оценки температурной эволюции эффективного поля в межгранульной среде. Зависимости $M(T)$ в условиях ZFC (аналогично зависимостям $R(T)$, рис. 6) приведены на рис. 7 (нижняя часть рисунка). Рис. 8 иллюстрирует температурные зависимости эффективного поля в межгранульной среде, полученные по выражению (3) с учетом данных $M(T): B_{\text {eff }}(T)=-4 \pi M(T) \alpha+H$. При расчете этих зависимостей значения параметра $\alpha$ брались теми же, что и для построения зависимостей $B_{\mathrm{eff}}(H)$ (рис. 4): $\alpha=10$ для $\mathbf{H}\|\mathbf{j}\| \mathbf{L}$ и $\alpha=22$ для $\mathbf{H} \perp \mathbf{j}$. При таком рассмотрении видно, что основное различие в поведении

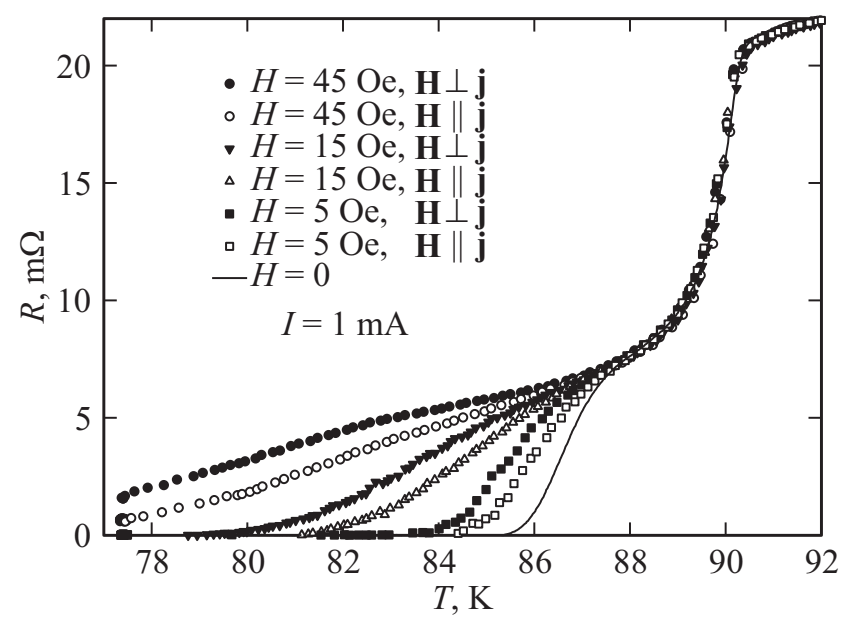

Рис. 6. Зависимости $R(T)$ в разных внешних полях при различной ваимной ориентации $\mathbf{H}$ и $\mathbf{j}$ в области резистивного перехода (при условиях ZFC).

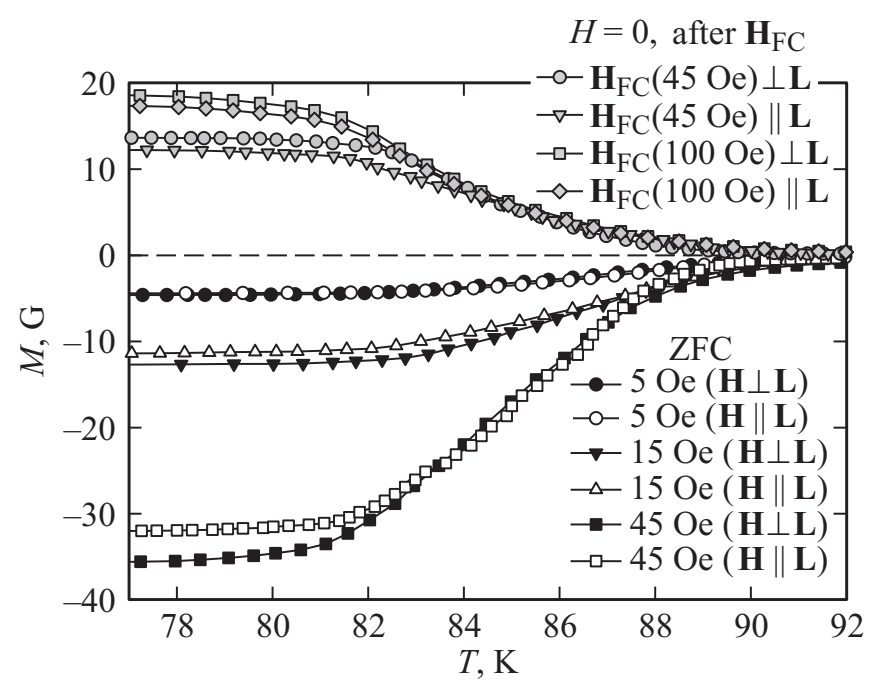

Рис. 7. Температурные зависимости магнитного момента $M(T)$ исследованного образца в разных внешних полях при различных взаимных ориентациях $\mathbf{H}$ и $\mathbf{L}$ (см. рис. $1, b)$ в условиях ZFC (нижняя часть рисунка, отрицательные значения $M$ ). Верхняя часть рисунка (положительные значения $M$ ) - зависимости $M(T)$, полученные в условиях FC с последующим „выключением“ внешнего поля $(H=0)$. 


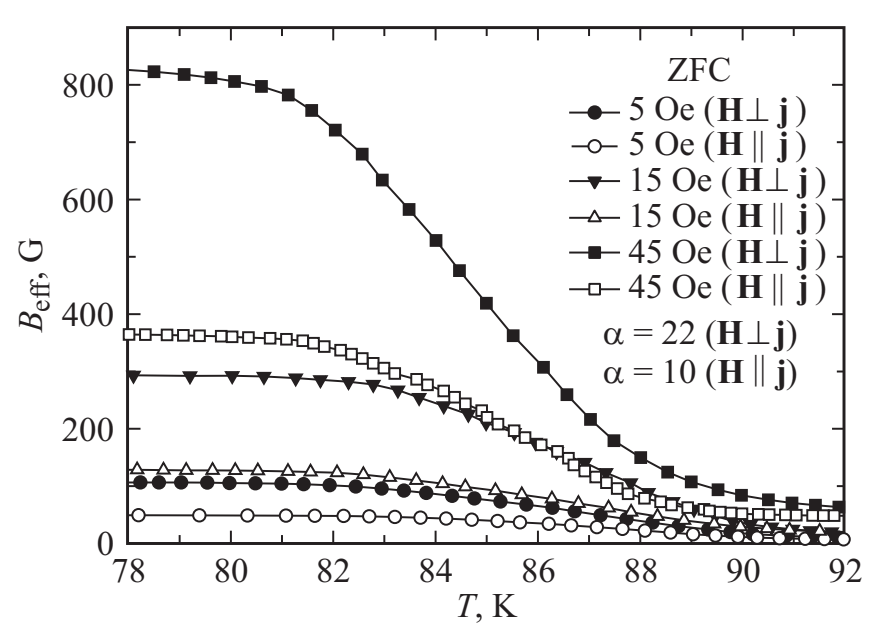

Рис. 8. Температурные зависимости эффективного поля в межгранульной среде для различных взаимных ориентаций $\mathbf{H}$ и $\mathbf{j}$, полученные при двух значениях $\alpha$ на основе данных $M(T)$ для условий ZFC, приведенных на рис. 7.

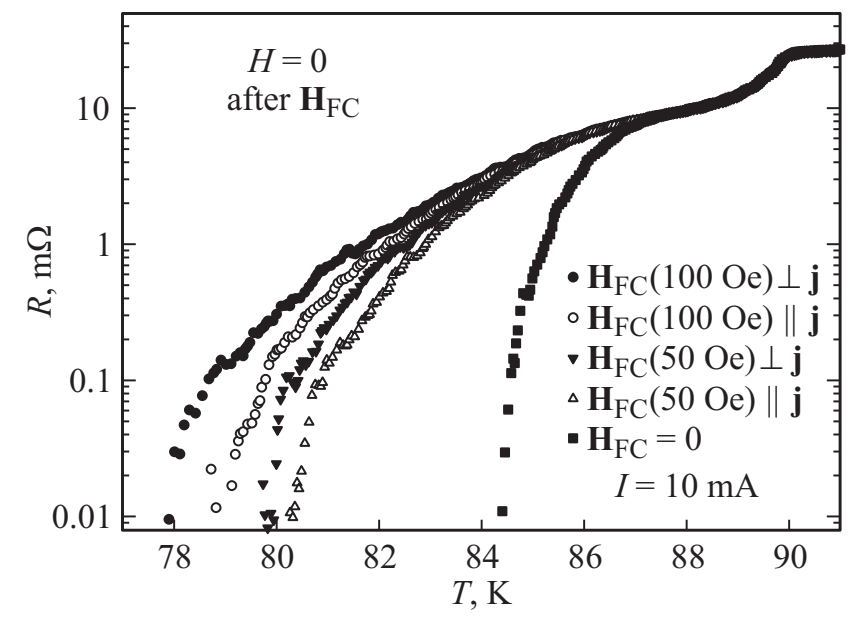

Рис. 9. Зависимости $R(T)$ исследованного образца в нулевом внешнем поле в области резистивного перехода. Охлаждение проводилось во внешнем поле при различных взаимных ориентациях Н и ј, после этого внешнее поле „выключалось“.

резистивного перехода обусловлено разной величиной эффективного поля в межгранульной среде, которое достаточно монотонно меняется с увеличением внешнего поля и дополнительно с изменением ориентации от параллельной к перпендикулярной.

Известно, что после выполнения процедуры FC образец обладает отрицательным магнитным моментом, но меньшим по модулю, чем при условиях ZFC. Затем, после выключения поля, магнитный момент образца принимает положительное значение ввиду захваченного потока. Зависимости $M(T)$, измеренные в нулевом внешнем поле (после выполнения условий $\mathrm{FC}$ ), приведены на рис. 7 (верхняя часть рисунка). После такой термомагнитной предыстории распределение индуцированных полей в межгранульной среде будет аналогично пока- занным на рис. $1, b$ (для условий охлаждения в $\mathbf{H}_{\mathrm{FC}} \perp \mathbf{j}$ ) и рис. $1, c$ (для $\mathbf{H}_{\mathrm{FC}} \| \mathbf{j}$ ), с той разницей, что внешнего поля уже нет $(H=0)$, а направления векторов $\mathbf{B}_{\text {ind }}$ и $\mathbf{M}_{G}$ сменились на противоположные (что уже неважно для протекания тока).

В рассматриваемом случае, так же как и для зависимостей $R(H)$ и $R(T)$ в условиях ZFC, будет различие между зависимостями $R(T)$ для условий $\mathbf{H}_{\mathrm{FC}} \perp \mathbf{j}$ и $\mathbf{H}_{\mathrm{FC}} \| \mathbf{j}$. Рис. 9 иллюстрирует это поведение. Все данные, приведенные на рис. 9, получены в нулевом внешнем поле, хотя термомагнитная предыстория была различной. Аналогичное поведение зависимостей $R(T)$ наблюдается и после выполнения условий ZFC с последующим „включением-выключением“ ${ }^{6}$ внешнего поля $\mathbf{H}_{\text {app }}=500$ Ое (не показано).

Следует отметить, что различие между зависимостями $R(T)$ для условий $\mathbf{H}_{\mathrm{FC}} \perp \mathbf{j}$ и $\mathbf{H}_{\mathrm{FC}} \| \mathbf{j}$ не столь значимое (рис. 9), как для условий $\mathbf{H}_{\mathrm{ZFC}} \perp \mathbf{j}$ и $\mathbf{H}_{\mathrm{ZFC}} \| \mathbf{j}$ (рис. 6), и отчетливо проявляется только в логарифмическом масштабе по $R$. Видимо, это связано с невыполнением условия $\alpha=$ const (см. раздел 2) для столь широкого диапазона полей. Действительно, между экспериментальными и расчетными зависимостями $\Delta H\left(H_{\downarrow}\right)$ (рис. 5) наблюдается расхождение в области полей $H_{\downarrow}$, меньших $\sim 100$ Ое. Минимумы экспериментальных зависимостей $R\left(H_{\downarrow}\right)$ (вставка на рис. 3) наблюдаются при значениях $H_{\downarrow}$, равных $\sim 25$ и $\sim 18$ Ое для $\mathbf{H} \perp \mathbf{j}$ и $\mathbf{H} \| \mathbf{j}$ соответственно. При $\alpha=22(\mathbf{H} \perp \mathbf{j})$ и $\alpha=10$ $\left(\mathbf{H} \| \mathbf{j}\right.$ ) зависимости $B_{\text {eff }}\left(H_{\downarrow}\right)$ (рис. 4) демонстрируют минимумы при бо́льших значениях $H_{\downarrow}(\sim 45$ и $\sim 40$ Ое $)$. Для того чтобы привести в соответствие минимумы зависимостей $B_{\text {eff }}\left(H_{\downarrow}\right)$ с минимумами зависимостей $R\left(H_{\downarrow}\right)$, в рамках рассматриваемого подхода необходимо использовать меньшие значения $\alpha(\sim 2.2$ и $\sim 1.5$ для ориентаций $\mathbf{H} \perp \mathbf{j} \quad$ и $\quad \mathbf{H} \| \mathbf{j} \quad$ соответственно). Условия термомагнитной предыстории для режима $H \rightarrow+H_{\text {app }} \rightarrow H=0$ (как и для зависимостей $R(H)$ ) и режима FC с последующим выключением поля (рис. 9) аналогичны, и, согласно модели критического состояния, захваченный поток остается преимущественно в центре гранул. Естественно, что для этих случаев влияние захваченного потока на межгранульную среду будет сказываться меньше, чем для случая, когда экранирующие токи протекают преимущественно вблизи поверхности гранул (при увеличении внешнего поля $H=H_{\uparrow}$ после условий ZFC). Это объясняет меньшее влияние сжатия потока в области малых полей (при $H=H_{\downarrow}$ ) и соответственно меньшие значения параметра $\alpha$. Тем не менее различие между случаями $\mathbf{H}_{\mathrm{FC}} \perp \mathbf{j}$ и $\mathbf{H}_{\mathrm{FC}} \| \mathbf{j}$ имеет место даже в нулевом внешнем поле, что иллюстрирует рис. 9, и оно связано с влиянием захваченного потока в гранулах на межгранульную среду.

\section{5. Заключение}

Таким образом, в работе продемонстрировано, что наблюдаемая анизотропия (магнитное поле-транспортный 
ток) магниторезистивных свойств гранулярных ВТСП вызвана сжатием магнитного потока в межгранульной среде. При различных взаимных ориентациях Н и $\mathbf{j}$ транспортный ток преимущественно „выбирает“ траектории, при которых туннелирование между гранулами происходит вдоль направления, соответствующего наименьшему углу между $\mathbf{B}_{\text {ind }}$ и $\mathbf{j}$, где силовые линии магнитной индукции наиболее разрежены. Для параллельной конфигурации $(\mathbf{H} \| \mathbf{j})$ величина параметра, характеризующего эффективную степень сжатия магнитного потока, примерно в 2 раза меньше, чем для перпендикулярной $(\mathbf{H} \perp \mathbf{j})$, и это выполняется для достаточно большого диапазона полей (от $\sim 10^{2}$ до $\left.\sim 10^{3} \mathrm{Oe}\right)$. При дальнейшем увеличении внешнего поля (либо при приближении температуры к $T_{C}$ ) влияние магнитных моментов гранул на межгранульную среду становится меньше, и в выражении (2) второе слагаемое становится больше первого. При этом наблюдаемая анизотропия магнитосопротивления уменьшается, т.е. величина $R(\mathbf{H} \| \mathbf{j}) / R(\mathbf{H} \perp \mathbf{j})$ приближается к единице в соответствии с модельными представлениями, предложенными в работе [7].

Авторы благодарят М.И. Петрова, К. Терентьева за приготовление образцов, А. Красикова и А. Фрейдмана за помощь в работе, А. Дубровского за обсуждение результатов.

\section{Список литературы}

[1] D. Lopez, F. de la Cruz. Phys. Rev. B 43, 13, 11478 (1991).

[2] D. Lopez, R. Decca, F. de la Cruz. Solid State Commun. 79, 11, 959 (1991).

[3] D. Lopez, R. Decca, F. de la Cruz. Supercond. Sci. Technol. 5, 5, 276 (1992).

[4] M.M. Asim, S.K. Hasanin. Solid State Commun. 80, 9, 719 (1991).

[5] A. Kilic, K. Kilic, S. Senoussi, K. Demir. Physica C 294, 203 (1998).

[6] O.V. Gerashchenko, S.L. Ginzburg. Supercond. Sci. Technol. 13, 332 (2000).

[7] D. Daghero, P. Mazzetti, A. Stepanescu, P. Tura. Phys. Rev. B 66, 13, 11478 (2002).

[8] А.А. Суханов, В.И. Омельченко. ФНТ 29, 4, 396 (2003).

[9] Д.А. Балаев, А.Г. Прус, К.А. Шайхутдинов, М.И. Петров. Письма в ЖТФ 32, 15, 67 (2006).

[10] D.A. Balaev, A.G. Prus, K.A. Shaukhutdinov, D.M. Gokhfeld, M.I. Petrov. Supercond. Sci. Technol. 20, 495 (2007).

[11] В.В. Деревянко, Т.В. Сухарева, В.А. Финкель. ФТТ 46, 10 , 1740 (2004)

[12] В.В. Деревянко, Т.В. Сухарева, В.А. Финкель. ФТТ 49, 10 , 1744 (2007)

[13] Т.В. Сухарева, В.А. Финкель. ФТТ 50, 6, 961 (2008).

[14] J. Bardeen, M.J. Stephen. Phys. Rev. 140, A1197 (1965).

[15] W.K. Kwok, U. Welp, G.W. Crabtree, K.G. Vandervoot, R. Hulscher, J.Z. Liu. Phys. Rev. Lett. 64, 8, 966 (1990).

[16] D.A. Balaev, S.I. Popkov, E.I. Sabitova, S.V. Semenov, K.A. Shaykhutdinov, A.V. Shabanov, M.I. Petrov. J. Appl. Phys. 110, 093918 (2011).
[17] Д.А. Балаев, С.В. Семенов, М.И. Петров. ФТТ 55, 12, 2305 (2013).

[18] D.A. Balaev, S.V. Semenov, M.I. Petrov. J. Supercond. Nov. Magn. 27, 1425 (2014).

[19] Д.А. Балаев, С.И. Попков, К.А. Шайхутдинов, М.И. Петров, Д.М. Гохфельд. ФТТ 56, 8, 1492 (2014).

[20] Д.А. Великанов. Патент РФ № 2339965. Опубл. 27.11.2008, Бюл. № 33.

[21] Д.М. Гохфельд. ФТТ 56, 12, 2298 (2014).

[22] Д.А. Балаев, Д.М. Гохфельд, А.А. Дубровский, С.И. Попков, К.А. Шайхутдинов, М.И. Петров. ЖЭТФ 132, 1340 (2007).

[23] Д.А. Балаев, А.А. Дубровский, К.А. Шайхутдинов, С.И. Попков, Д.М. Гохфельд, Ю.С. Гохфельд, М.И. Петров. ЖЭТФ 135, 271 (2009).

[24] Д.А. Балаев, А.А. Дубровский, С.И. Попков, Д.М. Гохфельд, С.В. Семенов, К.А. Шайхутдинов, М.И. Петров. ФTT 54, 11, 2027 (2012).

[25] В.В. Деревянко, Т.В. Сухарева, В.А. Финкель, Ю.Н. Шахов. ФТТ 56, 4, 625 (2014).

[26] Д.А. Балаев, А.А. Быков, С.В. Семенов, С.И. Попков, А.А. Дубровский, К.А. Шайхутдинов, М.И. Петров. ФТТ 53, 5, 865 (2011). 